$\begin{array}{cl}\text { PRAMANA } & \text { (C) Indian Academy of Sciences } \\ \begin{array}{c}\text { journal of } \\ \text { physics }\end{array} & \begin{array}{l}\text { Vol. } 72, \text { No. } 1 \\ \text { January } 2009 \\ \text { pp. } 239-250\end{array}\end{array}$

\title{
Physics beyond Standard Model: Working group 3 report
}

\author{
Coordinators: MONORANJAN GUCHAIT ${ }^{1}$ and SUDHIR K VEMPATI VE,* $^{2, *}$ \\ ${ }^{1}$ Tata Institute of Fundamental Research, Mumbai 400 001, India \\ ${ }^{2}$ Centre for High Energy Physics, Indian Institute of Science, Bangalore 560 012, India \\ ${ }^{*}$ Corresponding author \\ E-mail: guchait@mailhost.tifr.res.in; vempati@cts.iisc.ernet.in
}

Participants: Neelima Agarwal, S K Agarwalla, C S Aulakh, A Belyaev, S S Biswal, B Bhattacharjee, G Bhattacharyya, L Calibbi, D Choudhury, E J Chun, D Das,

A De Roeck, N G Deshpande, E Dudas, A Giri, D Grellshceid, R Godbole,

S Goswami, M Guchait, M Hirsch, R Kaul, B Kodrani, M C Kumar, A Kundu, Y Mambrini, P Mathews, B Mellado, R Mohanta, S Mohanty, A Nyffeler,

S Pakvasa, M K Parida, M Passera, C Petridou, S Poddar, P Poulose,

A Rajaraman, G Rajasekaran, V Ravindran, Kumar Rao, D P Roy, Probir Roy,

K A Saheb, V H Satheeshkumar, T Schwetz, A Tripathi, R Vaidya and S Vempati

\begin{abstract}
This is a summary of the beyond the Standard Model (including model building) working group of the WHEPP-X workshop held at Chennai from January 3 to 15, 2008.
\end{abstract}

Keywords. Beyond Standard Model; supersymmetry; little Higgs; GUTs; extra dimensions; technicolour.

PACS No. 12.60.-i

\section{Introduction}

Physics beyond Standard Model is a broad field which covers several established frameworks like supersymmetry, extra dimensions, technicolour, etc., and also new models of electroweak symmetry breaking like Higgsless models, new physics like unparticles, neutrino mass models and of course with the LHC expected to start giving the data before the next WHEPP, it was interesting to study the possible collider signatures of various models. Given this mandate, we thus had large overlaps with all the other working groups and in fact, most of our activities, either discussion sessions or informal talks, have been held jointly with either one or sometimes two other working groups. While it was difficult to cover all the above topics in the 12-day workshop, efforts were made to cover as much ground 


\section{Monoranjan Guchait and Sudhir K Vempati}

as possible. An overview of the status of the Standard Model and various frameworks of physics beyond Standard Model pertaining to Higgs and new methods of electroweak symmetry breaking was given by Gautam Bhattacharyya [1].

Supersymmetry still ranks as one of the most preferred physics beyond Standard Model as in addition to protecting Higgs mass, it has several nice features of being calculable, contains a natural dark matter candidate and the gauge coupling unification. In the recent years, there have been developments in string theory where it has been shown that all the moduli fields have been stabilised. Moduli fields can be thought of hidden sector fields where supersymmetry is spontaneously broken. The string solution for the moduli stabilization, namely, the KKLT framework provides novel relations between soft terms for the supersymmetric partners such as equality of gaugino masses at intermediate scale or mirage mediation. The phenomenology of the KKLT-type models has been covered by Yann Mambrini [2]. However, the situation can be more generic. Emilian Dudas [3] has summarized all the basic conditions required for consistent supersymmetric breaking. He then went on to elaborate on the novel ideas of hybrid scenarios where two mechanisms of supersymmetry breaking, for example, gauge mediation and gravity mediation or gauge mediation and anomaly mediation can co-exist and contribute equally leading to different kinds of patterns for soft terms which can hopefully be explored at colliders.

On the other hand, complementarity issues between indirect tests of supersymmetry using dark matter and flavour versus the direct experimental detection at LHC was also featured in several discussions during this workshop. D P Roy has summarized the issue of dark matter in supersymmetric theories, particularly concentrating on the non-universal gaugino mass scenario. Lorenzo Calibbi has reported on how dark matter regions are completely modified within SUSY grand unified theories sometimes leading to upper bounds and can possibly be easily distinguished from non-GUT models at a collider.

Flavour processes are still one of the major indirect search strategies for new physics. However, new physics can contribute to flavour physics even if it does not contain additional flavour structures, i.e., no new flavour structures other than the CKM in Standard Model. Such a scenario goes under the name minimal flavour violation (MFV). Rajaraman [4] reviewed the MFV formalism, its strengths and mentioned its pitfalls. He then proposed a modified version of this scheme where maximal flavour violation can occur in the new physics sector consistent with present day constraints. Perhaps the most stringent indirect test which any new physics model should pass would be the muon $g_{\mu}-2$. Massimo Passera has reviewed the status of $g_{\mu}-2$ within the Standard Model and the intricacies and complications involved in the computations of various contributions to it [5]. He then showed further that the present data give meaningful (upper) bounds on the Higgs mass which were surprisingly stringent and point towards possible resolution of the muon $g_{\mu}-2$ discrepancy in terms of the new physics. The related question of EDMs within supersymmetric theories has been reviewed by Rishikesh Vaidya and emphasis has been made on additional operators which can contribute significantly when $R$-parity is not conserved. Spontaneous CP violation in two-Higgs doublet models and implications for recent $B$ - and $D$-meson results was presented by Bhavik Kodrani. 
Neutrino masses within supersymmetry and other extensions of the Standard Model was reviewed by Chun [6]. After reviewing the standard see-saw model and leptogenesis scenario, Chun reviewed various alternative scenarios for the generation of neutrino masses and leptogenesis. In supersymmetric theories, simple no-scale boundary conditions (except for the Higgs) can lead to a nice synergy between flavour violation and leptogenesis. Triplet models and their signatures at LHC, emphasizing on the impact of the $\mathrm{CP}$ violating phases has been discussed by Thomas Schwetz. A simple model has been proposed by Chen as a solution to the question of how to incorporate the non-Abelian symmetry $A_{4}$ within a grand unified theory as it has been known that the $A_{4}$ symmetry would have difficulties in explaining the quark masses. Within supersymmetric grand unified theories, GUT thresholds play an important role, so much so that they can have strong impact on the intermediate scale which is present in $S O(10)$ models. This aspect has been emphasized by Parida in a discussion session.

With the LHC around the corner, most of the activity in the community is presently focused on computing various processes relevant for LHC. Keeping this in mind, there was a tutorial held by D Grellshceid on HERWIGG++. C Petridou then gave a welcoming talk on how the first signals from LHC would be like. A discussion was also held about unparticle physics led by A Rajaraman. Andreas Nyffeler had a detailed discussion about little Higgs models and their signatures at LHC and other colliders. Bruce Mellado discussed about what we could learn from studying the $4 l+E_{\mathrm{T}}$ signature. Both these discussions have led to intense discussions between theorists and experimentalists leading to the production of a dictionary of LHC signatures [7]. Further, Rohini Godbole reviewed anomalous $V V H$ and $V V V$ couplings (where $V$ stands for one of the SM gauge boson) and how one could constrain them from collider studies. Sudhansu Biswal presented the possible studies one can conduct at a linear collider if such couplings exist.

Finally new class of physics beyond Standard Models were also discussed during the workshop. Unparticles received quite a bit of attention. N G Deshpande presented a working group talk in which he reviewed the unparticle physics ideas and then concentrated on various constraints from both laboratory experiments and astrophysical sources. Strongly interacting light Higgs was also discussed a few times, and questions were asked whether little Higgs models could act as their UV completions.

In the following sections, we present summaries of working group talks (only those not presented separately in these proceedings) and further of discussions held and work conducted. We have organized it as follows : In $\S 2$, we cover supersymmetry. In $\S 3$, we will cover neutrino mass models, $\S 4$ deals with colliders and BSM physics. Section 5 deals with flavour and BSM physics and we close with some remarks and outlook.

\section{Supersymmetry}

2.1 Mixed neutralino DM in non-universal gaugino mass models

D P Roy (TIFR, Mumbai) 


\section{Monoranjan Guchait and Sudhir K Vempati}

In SUGRA models, the gaugino masses at the GUT scale arise from the VEV of the F term of the chiral superfield, responsible for SUSY breaking. Since the gauginos belong to the adjoint representation of the GUT group, symmetry consideration requires this chiral superfield to belong to the symmetric product of two adjoint representations, i.e. $24 \times 24=1+24+75+200$ for the $S U(5)$ case. In the mSUGRA model this is assumed to be a singlet. This leads to a bino LSP over most of the mSUGRA parameter space, which in turn leads generically to an over-abundance of DM relic density. The same is true for the 24-plet superfield. On the other hand, for 75 and 200-plet superfields, one generically gets higgsino dominated LSP, leading to under-abundance of DM relic density. We shall consider two SUSY models, where SUSY breaking takes place via admixtures of two superfields belonging to the $1+75$ and $1+200$ representations of $S U(5)$, with a single parameter alpha determining their relative contributions in each case. We shall see that with suitable values of alpha one gets a mixed bino-higgsino DM for the $1+75$ model and a mixed binowino DM for the $1+200$ model, with cosmologically compatible relic density in either case. Moreover, with the parameter alpha, so determined to give a mixed neutralino DM with cosmologically compatible relic density, each of the two models is as predictive as the mSUGRA model. So one expects to get fairly precise and unambiguous predictions of the resulting SUSY signals in LHC and DM experiments for both the models.

\subsection{Dark matter and collider}

A Belayev (University of Southampton, UK), U Chattopadhyay (IACS, Kolkata) and D P Roy (TIFR, Mumbai)

The minimal supersymmetric Standard Model (MSSM) offers the lightest supersymmetric particle (LSP) assumed to be the lightest neutralino as a dark matter (DM) candidate. Since the DM candidate is expected to be neutral and mass $~ 100$ $\mathrm{GeV}$, it is an ideal candidate. In mSUGRA model, essentially there are two regions in $m_{0}-m_{1 / 2}$ plane, the focus point and stau co-annihilation region corresponding to very large and small $m_{0}$ values, respectively. The detection possibility of the corresponding signals at LHC and the linear collider was discussed in the context of various SUSY models. A problem was discussed, in which case neutralino contains both higgsino and gauginos, i.e mixed scenario case and it is being investigated by some authors.

\subsection{Neutralino dark matter in SUSY-SU(5) with RH neutrinos}

$$
\text { Lorenzo Calibbi (SISSA, Italy) }
$$

In the CMSSM there are only three regions of the parameter space which provide the correct relic density for neutralino DM and are not excluded by the LEP limits on the mass of the SUSY particles: (i) the $\tilde{\tau}$ co-annihilation region (where $m_{\tilde{\tau}_{1}} \simeq m_{\tilde{\chi}_{1}^{0}}$ ), (ii) the $A$-pole funnel region $\left(m_{A} \simeq 2 m_{\tilde{\chi}_{1}^{0}}\right)$ and (iii) the focus point, where there is 


\section{Physics beyond Standard Model}

an enhancement of the Higgsino component of $\tilde{\chi}_{1}^{0}$ (and so of the annihilation crosssection) for small values of $\mu$. In such regions the (co)annihilation cross-section of $\tilde{\chi}_{1}^{0}$ is enhanced and the DM relic density does not exceed the WMAP bound. All these WMAP-allowed regions require very special relations among the parameters. A simple extension of the CMSSM, namely an evolution of the parameters above the GUT scale $M_{\mathrm{GUT}}$ and/or the presence of heavy sterile $\mathrm{RH}$ neutrinos, can destabilize such critical relations and thus modify the phenomenology of neutralino DM. In a simple SUSY-GUT framework, based on $S U(5)$ with the addition of RH neutrino fields (with one $\mathcal{O}(1)$ neutrino Yukawa), the RGE running of the soft parameters is modified in such a way that the allowed parameter space is wider than in the CMSSM $[8,9]$. Since in the CMSSM two of the three DM branches listed above have a connection with excluded regions (namely the region where the LSP is the lightest $\tilde{\tau}$ and the region where REWSB does not take place), we can expect that $S U(5)_{\mathrm{RN}}$ significantly changes the $\tilde{\chi}_{1}^{0} \mathrm{DM}$ phenomenology. The modifications of the $S U(5)_{\mathrm{RN}}$ parameter space with respect to CMSSM are sufficient to offset the conditions which give viable dark matter in CMSSM at low $\tan \beta$. In fact, there are quite strong lower bounds on the neutralino mass and $\tan \beta[9]: \tan \beta \gtrsim 34 ; m_{\tilde{\chi}_{1}^{0}} \gtrsim 160 \mathrm{GeV}$. Moreover, the peculiar phenomenology of $\tilde{\tau}$ co-annihilation region determines an upper bound on the LSP mass (around $250-350 \mathrm{GeV}$ ) for some regions of the parameter space (small $A_{0}, \tan \beta \lesssim 45$ ). Such an upper bound can be useful to distinguish this scenario from the CMSSM at colliders. Finally, the $A$-pole funnel branch appears for very large $\tan \beta$, such as in CMSSM, while focus point is absent, as a consequence of the $S U(5)_{\mathrm{RN}}$ RGE enhancement of the Higgs parameter $\mu$. An interesting possibility is the study of the average polarization of $\tau$ leptons coming from $\tilde{\tau}$ decays, which is possible with good accuracy at the international linear collider (ILC) [10]. $\tau$-polarization gives a deep insight of the mixing structure of staus and neutralinos and should be able to distinguish $S U(5)_{\mathrm{RN}}$ from CMSSM as long as SUSY spectrum lies in the stau co-annihilation region [11].

\section{Neutrino mass models}

\section{$3.1 \mathrm{~A}$ TeV scale Higgs triplet and neutrino properties at LHC}

\section{Thomas Schwetz (CERN, Switzerland)}

A model for neutrino masses is discussed, where a Majorana mass term for neutrinos arises from the VEV of an $S U(2)$ Higgs triplet. Assuming that this triplet has a mass below the $\mathrm{TeV}$ scale it will be accessible at the LHC, and the decay of its doubly-charged component $H^{ \pm \pm}$into two like-sign leptons provides a very clean experimental signature. In this scenario, the branching of $H^{ \pm \pm}$into a lepton pair $\ell_{\alpha}^{ \pm} \ell_{\beta}^{ \pm}(\alpha, \beta=e, \mu, \tau)$ is proportional to the corresponding element of the neutrino mass matrix $\left|M_{\alpha \beta}\right|^{2}$. Therefore, an investigation of the $H^{ \pm \pm}$decays at LHC may allow us to determine the absolute neutrino mass scale, the type of the neutrino mass ordering, and Majorana phases in the neutrino mass matrix [12]. 


\title{
Monoranjan Guchait and Sudhir K Vempati
}

3.2 Tri-bimaximal neutrino mixing and CKM matrix from finite group ${ }^{(d)}{ }^{T}$ in $S U(5)$

\author{
Mu-Chun Chen (University of California, USA) and K T Mahanthappa
}

(Colarado University, USA)

A grand unified model based on $S U(5)$ combined with the double tetrahedral group is presented, ${ }^{(d)} T$, which successfully, for the first time, gives rise to near tribimaximal leptonic mixing as well as realistic CKM matrix elements for the quarks, simultaneously. Due to the presence of the $Z_{12} \times Z_{12}^{\prime}$ symmetry, only nine operators are allowed in the model, and hence the model is very predictive, the total number of parameters being nine in the Yukawa sector for the charged fermions and the neutrinos. In addition, it provides a dynamical origin for the mass hierarchy without invoking additional $U(1)$ symmetry. Due to the ${ }^{(d)} T$ transformation property of the matter fields, the $b$-quark mass can be generated only when the ${ }^{(d)} T$ symmetry is broken, which naturally explains the hierarchy between $m_{b}$ and $m_{t}$. The $Z_{12} \times Z_{12}^{\prime}$ symmetry, to a very high order, also forbids operators that lead to nucleon decays. We obtain the Georgi-Jarlskog relations for three generations. This inevitably requires non-vanishing mixing in the charged lepton sector, leading to correction to the tri-bimaximal mixing pattern. The model predicts non-vanishing $\theta_{13}$, which is related to the Cabibbo angle as, $\theta_{13} \sim \theta_{\mathrm{c}} / 3 \sqrt{2}$. In addition, it gives rise to a sum rule, $\tan ^{2} \theta_{\odot} \simeq \tan ^{2} \theta_{\odot, \mathrm{TBM}}-\frac{1}{2} \theta_{\mathrm{c}} \cos \beta$, which is a consequence of the Georgi-Jarlskog relations in the quark sector. This deviation could account for the difference between the experimental best fit value for the solar mixing angle and the value predicted by the tri-bimaximal mixing matrix [14].

\subsection{Dirac leptogenesis in extended nMSSM}

\section{E J Chun (KIAS, South Korea) and Probir Roy (SINP, Kolkata)}

It is shown [13] that a version of the nearly minimal supersymmetric Standard Model (nMSSM), extended only in the singlet sector to include the additional superfields of right-handed neutrinos and very heavy Dirac particles conserving $B-L$, admits a viable scenario for Dirac leptogenesis and naturally small Dirac neutrino masses. The origin of the $(B-L)$-conserving high singlet neutrino scale and the desired supersymmetry breaking terms is associated with dynamical supersymmetry breaking in the hidden sector. This work originated at WHEPP-X, is now completed and published.

\subsection{Minimally coupled third-generation right-handed neutrino}

\section{Calibbi (SISSA, Italy), E J Chun (KIAS, South Korea) and S K Vempati (IISc, Bangalore)}

It is shown that a minimally coupled right-handed neutrino can have consequences for leptogenesis while having minimum influence in the generation of neutrino masses and almost null observable consequences for lepton flavour violation. In 
fact, this scenario would be very similar to that of a decoupling right-handed neutrino where neutrino masses, lepton flavour violation and leptogenesis effectively arise from a $3 \times 2$ see-saw. However, the third right-handed neutrino with large neutrino Yukawa coupling could have implications on supersymmetric dark matter in mSUGRA models.

\subsection{Low intermediate scales for leptogenesis in SUSY SO(10) GUTs}

Swarup Kumar Majee (HRI, Allahabad), Mina K Parida (IOPB, Bhubaneswar), Amitava Raychaudhuri (HRI, Allahabad) and Utpal Sarkar (PRL, Ahmedabad)

A low intermediate scale within minimal supersymmetric $S O(10)$ GUTs is a desirable feature to accommodate leptogenesis. We explore this possibility in models where the intermediate gauge symmetry breaks spontaneously by (a) doublet Higgs scalars and also (b) by triplets. In both scenarios gauge coupling unification requires the scale of left-right symmetry breaking $\left(M_{\mathrm{R}}\right)$ to be close to the unification scale. This will entail unnaturally small neutrino Yukawa couplings to avoid the gravitino problem and allow successful leptogenesis. We point out that any one of the three options - threshold corrections due to the mass spectrum near the unification scale, gravity-induced non-renormalizable operators near the Planck scale, or presence of additional light Higgs multiplets - can permit unification along with much lower values of $M_{\mathrm{R}}$ as required for leptogenesis. In the triplet model, independent of these corrections, we find a lower bound on the intermediate scale, $M_{\mathrm{R}}>10^{9} \mathrm{GeV}$, arising from the requirement that the theory must remain perturbative at least up to the GUT scale. We show that in the doublet model $M_{\mathrm{R}}$ can even be in the TeV region which, apart from permitting resonant leptogenesis, can be tested at LHC and ILC [15].

\section{BSM physics at colliders}

\subsection{First physics at $\mathrm{LHC}$}

\section{Chara Petridou (CERN, Switzerland)}

The first beam proton-proton collison for LHC experiment at CERN, Geneva, Switzerland, is expected to take place at the end of this year 2008 with a luminosity less than $100 \mathrm{pb}^{-1}$. The complexity of this experiment is enormous as far as hardware and software are concerned. In the very beginning the main task will be to understand the behaviour of detector before going for any data analysis to look for new physics. In this view, in the first few years of running, the main engagement will be to validate the Standard Model (SM) by measuring the masses of known particles and cross-sections of known SM physics processes. Even with the low luminosity $\left(<100 \mathrm{pb}^{-1}\right)$, a huge number of events for SM processes are expected, e.g. about $10^{6} W$ and $10^{5} Z$ production with $W \rightarrow \mu \nu$ and $Z \rightarrow \mu \mu$, will be produced. Similarly, about $10^{4} t \bar{t}$ events will be produced. These huge number of initial SM 


\section{Monoranjan Guchait and Sudhir K Vempati}

events will be used to calibrate detectors and validate software, which will be used in probing unknown physics. Possibly, some hints of new physics may appear at the early phase, like $Z^{\prime}$ which is a new gauge boson of heavy mass $\sim \mathrm{TeV}$ and predicted by various BSMs. This gauge boson can be observed via resonance, a narrow mass peak on top of small Drell-Yann background, either through di-muon or di-electron channel even with low luminosity like $\sim 1 \mathrm{fb}^{-1}$. Interestingly, in the early data with $100 \mathrm{pb}^{-1}$ luminosity, one may be able to find hints of supersymmetry in jet plus missing transeverse energy channel, if the mass of sparticles, mainly squarks and gluinos are at the lower side $(<1 \mathrm{TeV})$.

\subsection{Studying anomalous VVH and VVV CP-violating couplings at LHC}

A Belyaev (University of Southampton, USA), S Bhattacharya (Delhi University), D Choudhury (Delhi University), R M Godbole (IISc, Bangalore), B Mellado

(University of Wisconsin, USA), C Petridou (CERN, Switzerland) and A Rajaraman (University of California, USA)

If the Higgs boson coupling with fermions contains an axial part, then CP conservation is not guaranteed in the decay of Higgs boson to fermions. Similarly, gauge boson $(V=W / Z)$ couplings with Higgs can have an induced CP non-conserving part via loops. In those cases it is interesting to study how the $\mathrm{CP}$ violating effects can be observed at LHC. It was shown that in the process of Higgs production and its subsequent decay $H \rightarrow f \bar{f}$ and $H \rightarrow Z Z\left(Z^{*}\right) \rightarrow f \bar{f} f^{\prime} \bar{f}^{\prime}$, by observing different angular distributions, invariant mass distributions and also constructing angular correlations among decay products, it may be possible to study the $\mathrm{CP}$ violating effects. Perhaps, by constructing some rate asymmetry, it is also possible to probe the CP violating couplings at LHC.

\subsection{Little Higgs models at colliders}

$$
\text { Andreas Nyffeler (HRI, Allahabad) }
$$

Little Higgs models $[16,17]$ have been proposed as a solution to the little hierarchy problem of the Standard Model, i.e., the tension between a light Higgs mass and the large scale of new physics of the order of a few $\mathrm{TeV}$ from electroweak precision tests. In little Higgs models, the Higgs is a pseudo-Goldstone boson of a symmetry breaking that takes place at around $1 \mathrm{TeV}$. It receives a small mass only through radiative effects and the underlying (broken) symmetry protects the Higgs mass from getting quadratic divergences at one loop, thereby removing the little hierarchy problem. In order to ease constraints from precision tests, one can further impose a discrete symmetry, $T$-parity [18-20], which allows a rather low scale of symmetry breaking $[21,22]$ and leads to new particles in the range of a few hundred $\mathrm{GeV}$ and a natural dark matter candidate.

In this talk, we reviewed the basic ideas of little Higgs models and some recent developments relevant for the physics at the LHC. I concentrated mainly on the 
littlest Higgs model with conserved $T$-parity, which is a weakly interacting alternative to the MSSM with conserved $R$-parity and can lead to similar signatures at the LHC: pair production of new particles, cascade decays to the lowest lying stable state and large missing transverse energy [19,23]. In particular, we showed how one can distinguish the littlest Higgs model with $T$-parity from the MSSM using trileptons, even in a situation when the mass spectrum of the littlest Higgs model resembles the supersymmetric pattern [24]. We also discussed some modifications of Higgs boson properties (production and decay) in this model [20], including a large invisible decay branching ratio of the Higgs boson, if the symmetry breaking scale is low [22].

\subsection{Role of polarization in probing anomalous gauge interactions of the Higgs}

Sudhansu S Biswal (IISc, Bangalore), Debajyoti Choudhury (Delhi University), Rohini M Godbole (IISc, Bangalore) and Mamta (Delhi University)

We explore the use of polarized $e^{+} / e^{-}$beams and/or the information on final state decay lepton polarizations in probing the interaction of the Higgs boson with a pair of vector bosons. A model-independent analysis of the process $e^{+} e^{-} \rightarrow f \bar{f} H$, where $f$ is any light fermion, is carried out through the construction of observables having identical properties under the discrete symmetry transformations as different individual anomalous interactions. This allows us to probe an individual anomalous term independent of the others. We find that initial state beam polarization can significantly improve the sensitivity to CP-odd $Z Z H$ couplings. Moreover, ability to isolate events with a particular $\tau$ helicity with even $40 \%$ efficiency, can improve sensitivities to the $\tilde{T}$-odd $Z Z H$ couplings by as much as a factor of 3 . In addition, the contamination from the $Z Z H$ vertex contributions present in the measurement of the $W W H$ couplings can be reduced to a great extent employing polarized beams. The effects of ISR and beamsstrahlung, which can be relevant for higher values of the beam energy are also included in the analysis.

\section{Flavour and BSM physics}

5.1 EDM of neutron, deuteron and mercury in supersymmetry without R-parity

Rishikesh Vaidya (BITS, Pilani)

$P$ and $T$ violating electric dipole moments (EDMs) of fermions, heavy atoms and molecules are interesting CP violating observables that provide essentially background-free and sensitive probes of physics beyond SM. Though the search for non-vanishing EDM has so far not yielded any results, the present experimental scenario with regard to EDM measurements is very encouraging, with most of them well within the range of interesting predictions from physics beyond SM. We presented striking one-loop contributions to the EDM (including the colour EDMs) of mercury, neutron and deuteron from supersymmetry without $R$-parity. Being 


\section{Monoranjan Guchait and Sudhir K Vempati}

proportional to top Yukawa and top mass, such contributions are often large, and since these are proportional to hitherto unconstrained combinations of bilinear and trilinear RPV parameters, they are all the more interesting. Dominant contributions come from the combinations $B_{i}^{*} l_{i j 1}^{\prime}$ for which we obtain robust bounds. It turns out that neutron and deuteron EDMs receive much stronger contributions than mercury EDM. Even if $R$-parity violating couplings are real, CKM phase does induce RPV contribution and for some cases such a contribution is as strong as contribution from phases in the $R$-parity violating couplings. Hence, we have bounds directly on $\left|B_{i}^{*} l_{i j 1}^{\prime}\right|$ even if the RPV parameters are all real. Interestingly, even if slepton mass and/or $\mu$ is as high as $1 \mathrm{TeV}$, it still leads to neutron EDM that is an order of magnitude larger than the sensitivity at Los Alamos experiment. Since the results are not much sensitive to $\tan \beta$, our constraints will survive even if other observables like $B_{s} \rightarrow \mu^{+} \mu^{-}$tighten the constraints on $\tan \beta[25]$.

5.2 Complex CKM matrix, spontaneous CP violation and generalized $\mu-\tau$ symmetry

Anjan S Joshipura (PRL, Ahmedabad) and Bhavik P Kodrani (PRL, Ahmedabad)

The multi-Higgs models having spontaneous CP violation (SPCPV) and natural flavour conservation (NFC) lead to a real CKM matrix $V$ contradicting current evidence in favour of a complex $V$. This contradiction can be removed by using a generalized $\mu^{-\tau}$ (called 23) symmetry in place of the discrete symmetry conventionally used to obtain NFC. If the 23 symmetry is exact then the Higgs-induced flavour changing neutral currents (FCNC) vanish as in the case of NFC. 23 breaking introduces SPCPV, a phase in $V$ and suppressed FCNC among quarks. The FCNC couplings $F_{i j}^{d, u}$ between $i$ and $j$ generations show a hierarchy $\left|F_{12}^{d, u}\right|<\left|F_{13}^{d, u}\right|<\left|F_{23}^{d, u}\right|$ with the result that the FCNC can have observable consequences in $B$-mixing without conflicting with the $K^{0}-\bar{K}^{0}$ mixing. Detailed fits to the quark masses and the CKM matrix are used to obtain the (complex) couplings $F_{i j}^{d}$ and $F_{i j}^{u}$. Combined constraints from flavour and CP violations in the $K, B_{d}, B_{s}, D$ mesons are analysed within the model. They allow (i) relatively light Higgs, 100-150 GeV, (ii) measurable extra contributions to the magnitudes and phases of the $B_{d, s}^{0}-\bar{B}_{d, s}^{0}$ mixing amplitudes and (iii) the $D^{0}-\bar{D}^{0}$ mixing at the current sensitivity level [26].

\section{New models}

\subsection{UV completion of little Higgs with technicolor}

G Bhattacharyya (SINP, Kolkata), D Choudhury (Delhi University) and R Kaul (IMSc, Chennai)

It is proposed that little Higgs models are valid up to $10 \mathrm{TeV}$. Above that scale technicolour takes over. Technifermions transform under an $S U(N)$ flavour group and $G_{\mathrm{TC}}$ gauge group. Strong dynamics form condensates at that scale $(\Lambda)$. We 


\section{Physics beyond Standard Model}

have investigated mechanism of how to avoid severe constraints from electroweak precision tests. A concrete formalism starting with an $S U(5)$ flavour group is realized.

\subsection{Long-range forces and limits on unparticle interactions}

\section{N G Deshpande (University of Oregon, USA)}

A review of unparticle physics was presented. Couplings between Standard Model particles and unparticles from a non-trivial scale invariant sector can lead to longrange forces. If the forces couple to quantities such as baryon or lepton (electron) number, stringent limits result from tests of the gravitational inverse square law. These limits are much stronger than from collider phenomenology and astrophysics $[27]$.

\section{Remarks and outlook}

The advent of the LHC era is perhaps the most exciting time for people working on particle physics, particularly, physics beyond Standard Model. Whatever be the results from $\mathrm{LHC}$, it is for sure going to alter the way physics beyond Standard Model is seen, understood or perceived currently. In the present workshop, we have tried to summarize various aspects of physics beyond Standard Model within some popular frameworks, especially concentrating on LHC physics. The discussions held during this workshop have already led to a couple of publications and more are in pipeline.

\section{References}

[1] G Bhattacharryya, These proceedings

[2] Y Mambrini, These proceedings

[3] Emilian Dudas, These proceedings

[4] A Rajaraman, These proceedings

[5] M Passera, These proceedings

[6] E J Chun, These proceedings

[7] A Belyaev et al, These proceedings

[8] L Calibbi, A Faccia, A Masiero and S K Vempati, Phys. Rev. D74, 116002 (2006), arXiv:hep-ph/0605139

[9] This talk was based on: L Calibbi, Y Mambrini and S K Vempati, J. High Energy Phys. 0709, 081 (2007), arXiv:0704.3518 [hep-ph]

[10] R M Godbole, M Guchait and D P Roy, Phys. Lett. B618, 193 (2005), arXiv:hep$\mathrm{ph} / 0411306$

[11] L Calibbi, R Godbole, Y Mambrini and S K Vempati, arXiv:0710.0726 [hep-ph]

[12] This talk was based on: J Garayoa and T Schwetz, Neutrino mass hierarchy and Majorana CP phases within the Higgs triplet model at the LHC, arXiv:0712.1453 [hep-ph], J. High Energy Phys., to appear 


\section{Monoranjan Guchait and Sudhir K Vempati}

[13] This work was published in: E J Chun and P Roy, J. High Energy Phys. 0806, 089 (2008), arXiv:0803.1720 [hep-ph]

[14] This talk was based on: M C Chen and K T Mahanthappa, Phys. Lett. B652, 34 (2007), arXiv:0705.0714 [hep-ph]

[15] This talk was based on: S K Majee, M K Parida, A Raychaudhuri and U Sarkar, Phys. Rev. D75, 075003 (2007), arXiv:hep-ph/0701109

[16] N Arkani-Hamed, A G Cohen and H Georgi, Phys. Lett. B513, 232 (2001)

N Arkani-Hamed, A G Cohen, T Gregoire and J G Wacker, J. High Energy Phys. 0208, 020 (2002)

N Arkani-Hamed, A G Cohen, E Katz and A E Nelson, J. High Energy Phys. 0207, 034 (2002)

[17] M Schmaltz and D Tucker-Smith, Ann. Rev. Nucl. Part. Sci. 55, 229 (2005) M Perelstein, Prog. Part. Nucl. Phys. 58, 247 (2007)

M C Chen, Mod. Phys. Lett. A21, 621 (2006)

E Accomando et al, hep-ph/0608079, Chapter 7

[18] H C Cheng and I Low, J. High Energy Phys. 0309, 051 (2003); J. High Energy Phys. 0408, 061 (2004)

I Low, J. High Energy Phys. 0410, 067 (2004)

[19] J Hubisz and P Meade, Phys. Rev. D71, 035016 (2005)

[20] C R Chen, K Tobe and C P Yuan, Phys. Lett. B640, 263 (2006)

[21] J Hubisz, P Meade, A Noble and M Perelstein, J. High Energy Phys. 0601, 135 (2006) M Asano, S Matsumoto, N Okada and Y Okada, Phys. Rev. D75, 063506 (2007)

[22] R S Hundi, B Mukhopadhyaya and A Nyffeler, Phys. Lett. B649, 280 (2007)

[23] C O Dib, R Rosenfeld and A Zerwekh, J. High Energy Phys. 0605, 074 (2006) A Freitas and D Wyler, J. High Energy Phys. 0611, 061 (2006)

A Belyaev, C R Chen, K Tobe and C P Yuan, Phys. Rev. D74, 115020 (2006)

L Wang, W Wang, J M Yang and H Zhang, Phys. Rev. D75, 074006 (2007)

M Carena, J Hubisz, M Perelstein and P Verdier, Phys. Rev. D75, 091701 (2007)

Q H Cao, C S Li and C P Yuan, hep-ph/0612243

S Matsumoto, M M Nojiri and D Nomura, Phys. Rev. D75, 055006 (2007)

D Choudhury and D K Ghosh, J. High Energy Phys. 0708, 084 (2007)

M M Nojiri and M Takeuchi, Phys. Rev. D76, 015009 (2007)

Q H Cao and C R Chen, Phys. Rev. D76, 075007 (2007)

[24] A Datta et al, Phys. Lett. B659, 308 (2008)

[25] This talk was based on: C C Chiou, O C W Kong and R D Vaidya, Phys. Rev. F76, 013003 (2007), arXiv:0705.3939 [hep-ph]

[26] This talk was based on: A S Joshipura and B P Kodrani, arXiv:0706.0953 [hep-ph]

[27] This talk is based on: N G Deshpande, S D H Hsu and J Jiang, Phys. Lett. B659, 888 (2008), arXiv:0708.2735 [hep-ph] 